\title{
2. Erscheinungsformen von Behinderung in einer alternden Gesellschaft: Zur Kompetenz von älteren Menschen mit geistiger Behinderung
}

\author{
Von Andreas Kruse
}

Einführung

1. Zur Definition von Kompetenz 33

2. Kompetenz bei älteren Menschen mit geistiger Behinderung 36

3. Behinderungen als Folge spezifischer Person-Umwelt-Interaktionen 39

4. Die infrastrukturelle Umwelt: Inwieweit spiegelt sich die Kompetenzorientierung in den spezifischen Angeboten wider? 42

5. In welcher Hinsicht lassen sich die Kompetenz und Lebensqualität durch die Entwicklung innovativer Konzepte fördern?

5.1. Zentrale Merkmale der Kompetenz und Lebensqualität bei Behinderung im Alter aus einer Interventionsperspektive

5.2. Zentrale Kategorien innovativer Konzepte der Behindertenhilfe

5.3. Entwicklung innovativer Konzepte aus der Perspektive der Institutionen

6. Entwicklungsperspektiven 


\section{Einführung}

Die Lebenserwartung von Menschen mit geistiger Behinderung ist um fünf Jahre geringer als die der Normalbevölkerung, Menschen mit Down-Syndrom haben eine um zehn Jahre geringere Lebenserwartung als Menschen mit geistiger Behinderung und eine um 15 Jahre geringere Lebenserwartung als nicht behinderte Menschen (Haveman \& Stöppler, 2004 ${ }^{1}$; Yang, Rasmussen \& Friedman, 2002²). In den letzten Jahrzehnten hat eine Angleichung der durchschnittlichen Lebenserwartung von Menschen mit geistiger Behinderung und ohne Behinderung stattgefunden (SchulzNieswandt, $2006^{3}$ ). Auch wenn Menschen mit Down-Syndrom eine um etwa 15 Jahre geringere Lebenserwartung zeigen, so trifft doch der Anstieg der Lebenserwartung gerade auf diese Personengruppe zu: Im Zeitraum von 1950 bis heute hat sich deren durchschnittliches Lebensalter fast vervierfacht, nämlich von 15 Jahren auf fast 60 Jahre. Der medizinische Fortschritt, hier vor allem die Antibiotika-Therapie, die Behandlung von Infektionskrankheiten, die korrigierende Herzchirurgie, sowie Bildungs- und Rehabilitationskonzepte haben zu dieser positiven Entwicklung beigetragen (Driller \& Pfaff, 2006 ${ }^{4}$ ). Die Definition von Grenzen der Lebenserwartung für Menschen mit geistiger Behinderung erscheint vor dem Hintergrund der Angleichung der Lebenserwartung nicht mehr als angemessen (Haveman \& Stöppler, $2004^{5}$ ).

Ergebnisse wissenschaftlicher Untersuchungen zur Kompetenz und Kompetenzförderung bei Menschen mit geistiger Behinderung deuten auf Plastizität und Veränderungskapazität auch im mittleren und hohen Erwachsenenalter (Ding-Greiner \& Kruse, $2004^{6}$ ). Aus diesem Grunde ist es notwendig, in den Konzepten der Begleitung und Betreuung älterer Menschen mit geistiger Behinderung zum einen den Bildungsaspekt zu akzentuieren (Kraft, 2006 ), zum anderen den Umweltaspekt, wobei hier der anregende, ressourcenförderliche Charakter der Umwelt im Zentrum steht $\left(\right.$ Wacker \& Wahl, $\left.2007^{8}\right)$.

$1 \quad$ Haveman/Stöppler (2004), Altern mit geistiger Behinderung. Grundlagen und Perspektiven für Begleitung, Bildung und Rehabilitation, Stuttgart: Kohlhammer.

2 Yang. et al. (2002), Mortality associated with Down's Syndrome in the USA, Lancet, 359, 10191025.

3 Schulz-Nieswandt (2006), Alternsformen, Lebenserwartung und Altersstruktur behinderter Menschen - unter besonderer Berücksichtigung angeborener Formen geistiger Behinderung, in: Krueger./Degen (Hrsg.), Das Alter behinderter Menschen (S. 142-201), Freiburg: Lambertus.

4 Driller/Pfaff (2006), Soziodemografische Struktur von Menschen mit Behinderung in Deutschland, in: Krueger/Degen (Hrsg.), Das Alter behinderter Menschen (S. 26-117), Freiburg: Lambertus.

5 Haveman/Stöppler (2004), Altern mit geistiger Behinderung. Grundlagen und Perspektiven für Begleitung, Bildung und Rehabilitation, Stuttgart: Kohlhammer.

6 Ding-Greiner/Kruse (2004), Alternsprozesse bei Menschen mit geistiger Behinderung, in: Kruse/Martin (Hrsg.), Enzyklopädie der Gerontologie (S. 519-532), Bern: Huber.

$7 \quad$ Kraft (2006), Pflege älterer Menschen mit Behinderungen: Tendenzen, Entwicklungen, Perspektiven, in: Krueger/Degen (Hrsg.), Das Alter behinderter Menschen (S. 192-206), Freiburg: Lambertus.

8 Wacker/Wahl (2007), Altersfreundliche und ressourcenförderliche Umwelten, in: Bertelsmann Stiftung (Hrsg.), Alter neu denken (S. 217-247), Gütersloh: Verlag Bertelsmann Stiftung. 
Aus diesem Grunde ist ausdrücklich nachfolgender Aussage zuzustimmen:

„Die Eingliederungshilfe mit ihrem ganzheitlichen Anspruch geht also weit über die Hilfe zur Pflege hinaus, weil sie auch Leistungen der Rehabilitation und Förderung umfasst. Es ist hier zunächst einmal von zentraler Bedeutung sowohl für die fachliche Weiterentwicklung wie auch für die Refinanzierung der Pflege älterer behinderter Menschen, dass diese weiterhin im Wirkungskreis der Eingliederungshilfe verbleibt“" $\left(\right.$ Kraft, 2006, S.196 f. $\left.{ }^{9}\right)$.

\section{Zur Definition von Kompetenz}

Den Ausgangspunkt unserer Überlegungen bildet ein Verständnis von Kompetenz, das die Bedeutung der Umwelt für die Erhaltung der Leistungsfähigkeit des Menschen hervorhebt. Kompetenz umfasst dabei

„Fähigkeiten und Fertigkeiten des Menschen zur Erhaltung oder Wiederherstellung eines selbstständigen, selbstverantwortlichen und sinnerfüllten Lebens in einer anregenden, unterstützenden, zur selbstverantwortlichen Auseinandersetzung mit Anforderungen motivierenden sozialen, räumlichen und infrastrukturellen Umwelt“ (Kruse, 2007 ${ }^{10}$; Kruse \& Schmitt, $\left.2006^{11}\right)$.

Für die Behindertenhilfe kann sich eine derartige Definition von Kompetenz in dreifacher Hinsicht als hilfreich erweisen:

Erstens: In dieser Definition wird zwischen Selbstständigkeit, Selbstverantwortung und sinnerfülltem Leben differenziert. Die in der Literatur zum Alter vielfach anzutreffende Beschränkung auf die Selbstständigkeit (zum Beispiel im Sinne der selbstständigen Ausführung von Aktivitäten des täglichen Lebens) wird hier vermieden. Selbstständigkeit bildet zwar ein bedeutsames Merkmal der Kompetenz, doch keinesfalls das einzige Merkmal. Hinzu kommt die Selbstverantwortung des Menschen, die als Fähigkeit und Bereitschaft verstanden wird, das Leben in einer den eigenen Leitbildern eines guten Lebens (,Wer bin ich? Was will ich?“) folgenden Weise zu gestalten. In der Selbstverantwortung kommt nicht nur eine Fähigkeit zum Ausdruck (Reflektion eigenen Handelns), sondern auch eine Motivlage (Bereitschaft, Ziele zu definieren und diese zu verwirklichen). Dabei ist zu berücksichtigen, dass in der Behindertenhilfe nicht nur die Fähigkeiten, sondern eben auch die Motivlage des Menschen besondere Anforderungen an die Mitarbeiterinnen und Mitarbeiter stellt. Wie kann es - vor allem nach Ausscheiden des Menschen mit Behinderung aus der Werkstätte - gelingen, diesen dazu zu motivieren, neue Ziele zu definieren und sich verantwortlich mit den Entwicklungsaufgaben des höheren Lebensalters

9 Kraft. (2006), Pflege älterer Menschen mit Behinderungen: Tendenzen, Entwicklungen, Perspektiven, in: Krueger/Degen (Hrsg.), Das Alter behinderter Menschen (S. 192-206), Freiburg: Lambertus.

10 Kruse (2007), Veränderbarkeit geistiger und körperlicher Fähigkeiten im Alter, in: Brandtstädter/Lindenberger (Hrsg.), Entwicklungspsychologie (S. 622-649), Stuttgart: Kohlhammer

11 Kruse/Schmitt (2006), Adult education, in: Birren (Ed.), Encyclopedia of Gerontology (pp.312332), Oxford: Elsevier. 
auseinander zu setzen (vgl. Wacker, Metzler \& Trost, 1996 ${ }^{12}$ )? Diese Frage kann auch in anderer Weise ausgedrückt werden: Wie kann es gelingen, den Menschen dazu zu motivieren, seine Fähigkeiten (im kognitiven, im alltagspraktischen, im physischen Bereich) einzusetzen und aufrechtzuerhalten (vgl. Haveman, $2001^{13}$ )? In der angeführten Definition wird neben der Selbstständigkeit und der Selbstverantwortung die sinnerfüllte Lebensgestaltung als bedeutsames Merkmal der Kompetenz gewertet. Auch hier haben wir es nicht nur mit einer Fähigkeit, sondern zudem mit einer Motivlage zu tun: Sinnerfahrung ist nur möglich, wenn Menschen offen für den Aufforderungscharakter, den Anregungsgehalt einer Situation sind. Bei der sinnerfüllten Lebensgestaltung wird deswegen von einer Fähigkeit gesprochen, da Menschen zum einen in der Lage sein müssen, zu beurteilen, in welchen Lebenssituationen sich die Erfahrung der Stimmigkeit einstellen kann, da sie zum anderen über Techniken verfügen müssen, solche Situationen aktiv aufzusuchen oder herzustellen. Die Angebote zur Tagesstruktur - die ein zentrales Element der Behindertenhilfe darstellen - lassen sich im Kern von dem Ziel leiten, ein „sinnstiftendes Angebot" zu unterbreiten, welches zum Beispiel nach Ausscheiden aus der Werkstätte für Behinderte die frühere Berufstätigkeit zu ersetzen vermag (Hawkins, $1999^{14}$; Hessisches Sozialministerium \& Landeswohlfahrtsverband Hessen $2000^{15}$; Walker et al., $1999^{16}$ ).

Zweitens: In dieser Definition wird die große Bedeutung der Umwelt für die Fähigkeiten des Menschen zu einem selbstständigen, selbstverantwortlichen und sinnerfüllten Leben betont. Damit wird zum Ausdruck gebracht, dass die personzentrierte Definition von Behinderung - die ausschließlich die bei einem Menschen bestehenden Einschränkungen im Blick hat, hingegen nicht die möglichen Umweltbarrieren eines selbstständigen, selbstverantwortlichen und sinnerfüllten Lebens aufgegeben werden muss zugunsten einer Definition von Behinderung, welche die Wechselwirkungen zwischen Person und Umwelt akzentuiert. In der vorgeschlagenen Kompetenzdefinition wird hervorgehoben, dass die Umwelt - und zwar die räumliche, die soziale, die infrastrukturelle Umwelt - durch Anregungen, Unterstützung und Motivation (nämlich zur selbstverantwortlichen Auseinandersetzung mit Anforderungen und Aufgaben) einen substanziellen Beitrag zur Erhaltung und Wei-

12 Wacker/Metzler/Trost (1996), Förderung und das Recht auf Ruhestand: ein Spannungsverhältnis, in: Berghau/Knapic/Sievert (Hrsg.), Abschied vom Fürsorgedenken (S. 56-78), Köln: Kuratorium Deutsche Altershilfe.

13 Haveman (2001), Perspektiven der Integration älterer Menschen mit geistiger Behinderung, in: Hessisches Sozialministerium, Landeswohlfahrtsverband Hessen \& Bundesvereinigung Lebenshilfe für Menschen mit geistiger Behinderung e.V. (Hrsg.), Lebensräume älterer Menschen mit Behinderung (S. 157-180), Marburg: Lebenshilfe-Verlag.

14 Hawkins (1999), Rights, place of residence, and retirement: Lessons from case studies on aging, in: Herr/Weber (Eds.), Aging, rights, and quality of life (pp. 93-108), Baltimore: Brookes.

15 Hessisches Sozialministerium, Landeswohlfahrtsverband Hessen (2000), Lebensräume älterer Menschen mit Behinderung. Fachliche Leitlinien und Empfehlungen, Wiesbaden: Hessisches Sozialministerium.

16 Walker et al. (1999), Quality of life as a matter of human rights, in: Herr/Weber (Eds.), Aging, rights, and quality of life (pp. 109-132), Baltimore: Brookes. 
terentwicklung von Fähigkeiten leistet. Die in Beiträgen zur Pflegeforschung nachgewiesenen Zusammenhänge zwischen einer selbstständigkeitsfördernden Umwelt, die zur selbstständigen Ausführung von Aktivitäten des täglichen Lebens motiviert und die die stellvertretende Ausübung von Aktivitäten durch die soziale Umwelt vermeidet, sowie dem Gewinn an Selbstständigkeit im Pflegeprozess (Baltes, 1996) weist auf die große Bedeutung der Umwelt für die Erhaltung und Weiterentwicklung von Fähigkeiten sowie - umgekehrt - für die Vermeidung, Linderung oder Überwindung von Einschränkungen hin. Speziell im Hinblick auf die Erhaltung von Selbstständigkeit bei chronischen oder chronisch-progredienten Erkrankungen ist dem „Frailty-Konzept“ hoher theoretischer und praktischer Wert zuzuordnen (DingGreiner \& Lang, 2004 ${ }^{17}$ ). Dieses Konzept geht davon aus (und diese Annahme konnte auch empirisch gestützt werden), dass der Übergang von chronischen Erkrankungen zu Hilfebedarf oder Pflegebedarf auch durch die psychische Befindlichkeit sowie durch die räumliche und soziale Umwelt vermittelt ist (Rockwood et al., $2000^{18}$.

Drittens: In dieser Definition sind Merkmale der Person und der Umwelt aufgeführt, die eng mit den in der Behindertenhilfe entwickelten Bildungs- und Förderkonzepten verwandt sind. Die Eingliederungshilfe und die auf dieser gründenden heilpädagogischen Konzepte bleiben ausdrücklich nicht bei der Selbstständigkeit stehen, sondern sehen auch die Schaffung von Rahmenbedingungen für ein selbstverantwortliches und sinnerfülltes Leben als bedeutsame Aufgabe an. Dabei wird hervorgehoben, dass sich die Funktion der Umwelt nicht allein auf die Unterstützung des Menschen mit Behinderung beschränkt, sondern auch anregende und motivierende Aspekte einschließt. Verbindet man diese drei Funktionen der Umwelt mit den Fähigkeiten, die - der vorgeschlagenen Definition von Kompetenz zufolge kompetentes Handeln konstituieren (Selbstständigkeit, Selbstverantwortung, Sinnerfahrung), so ergeben sich hier zentrale Komponenten eines Bildungsbegriffs. Die Behindertenhilfe versteht den Einsatz ihrer Fördermaßnahmen auch als Beitrag zur lebenslangen Bildung des Menschen. Sie stellt auch in der Arbeit mit älteren behinderten Menschen die Frage nach Potenzialen (Theunissen, 2002 ${ }^{19}$ ), das heißt nach Fähigkeiten und Interessen, wie auch nach möglichen Ressourcen für die Gemeinschaft.

17 Ding-Greiner/Lang (2004), Alternsprozesse und Krankheitsprozesse - Grundlagen, in: Kruse/Martin (Hrsg.), Enzyklopädie der Gerontologie (S. 182-206), Bern: Huber.

18 Rockwood/Hogan/MacKnight et al. (2000), Conceptualization and measurement of frailty in elderly people, Drugs Aging, 17, 295-302.

19 Theunissen (2002), Die Stärken-Perspektive. Impulse für die pädagogische und therapeutische Arbeit mit Menschen, die als geistig behindert gelten, Geistige Behinderung, 3, 2002. 


\section{Kompetenz bei älteren Menschen mit geistiger Behinderung}

Zunächst seien einige Aussagen zur körperlichen Gesundheit und physischen Kompetenz bei älteren Menschen mit geistiger Behinderung getroffen. In Bezug auf die generelle Morbidität und Mortalität unterscheiden sich ältere Menschen mit geistiger Behinderung nicht von jenen ohne geistige Behinderung (Ding-Greiner \& Kruse, $2004^{20}$ ). Allerdings muss damit gerechnet werden, dass gesundheitliche Probleme bei älteren Menschen mit geistiger Behinderung und der daraus resultierende Versorgungsbedarf unterschätzt werden, da (a) Menschen mit Behinderung gesundheitliche Probleme möglicherweise seltener ausdrücken, (b) gesundheitliche Probleme bei ihnen mit einer noch höheren Wahrscheinlichkeit als Folge natürlicher Alternsprozesse oder von Behinderung interpretiert werden (eine falsche Interpretation, die vielfach Ursache von Unterversorgung ist) (Janicki et al., 2002 ${ }^{21}$ ). Zu den Barrieren des Zugangs älterer Menschen mit geistiger Behinderung zum medizinischen Versorgungssystem gehören vor allem folgende (Evenhuis et al., 200122):

a) Kommunikationsprobleme

b) Motorische Einschränkungen

c) Mangelnde Ausbildung und mangelndes Training von Ärzten und Pflegefachkräften

d) Zu hohe Kosten, zu hoher Zeitaufwand im Erleben der Angehörigen

e) Geringer sozialer Status von älteren Menschen mit Behinderung

Bei alten Menschen ohne Behinderung und alten Menschen mit geistiger Behinderung sind vergleichbare körperliche Erkrankungen erkennbar, wie HerzKreislauferkrankungen, Arthrosen, Frakturen mit anschließenden Funktionseinschränkungen, Diabetes mellitus Typ II und Lungenerkrankungen. Darüber hinaus ist bei Menschen mit geistiger Behinderung ähnlich wie bei Menschen ohne Behinderung ab dem 75. Lebensjahr das Risiko motorischer Einschränkungen, speziell aufgrund von Gon- und Coxarthrose, Parkinsonscher Erkrankung, Spondylitis und Osteoporose, erhöht. Seheinbußen und Hörbeeinträchtigungen sind bei Menschen mit geistiger Behinderung häufiger zu beobachten (Evenhuis, 1995 $\mathrm{a}^{23}, 1995 \mathrm{~b}^{24}$ ). Es besteht ein signifikanter Zusammenhang zwischen Alter einerseits sowie Hilfebedarf und Pflegebedarf andererseits, dieser Zusammenhang ist dabei unabhängig sowohl

20 Ding-Greiner/Kruse (2004), Alternsprozesse bei Menschen mit geistiger Behinderung, in: Kruse/Martin (Hrsg.), Enzyklopädie der Gerontologie (S. 519-532), Bern: Huber.

21 Janicki et al. (2002), Health characteristics and health services utilization in older adults with intellectual disability living in community residences, Journal of Intellectual Disability Research, 46, 287-298.

22 Evenhuis et al. (2001), Healthy ageing - adults with physical health issues, Journal of Applied Research in Intellectual Disabilities, 14, 175-194.

23 Evenhuis (1995a), Medical aspects of ageing in a population with intellectual disability: Visual impairment, Journal of Intellectual Disability Research, 39, 19-25.

24 Evenhuis (1995b), Medical aspects of ageing in a population with intellectual disability: Hearing impairment, Journal of Intellectual Disability Research, 39, 27-33. 
vom Vorliegen einer geistigen Behinderung als auch vom Grad der Behinderung (Maaskant et al., 1996 ${ }^{25}$ ). Während geistige Behinderungen allgemein keinen Einfluss auf Hilfe- und Pflegebedarf haben, lässt sich dieser für das Vorliegen von Down-Syndromen nachweisen: Bei Menschen mit Down-Syndrom sind die Funktionseinbußen signifikant stärker ausgeprägt als bei Menschen mit einer geistigen Behinderung, aber ohne Down-Syndrom.

Menschen mit geistiger Behinderung verfügen über geringere psychische und kognitive Ressourcen, die als Schutz gegen Belastungen im Alltag dienen können. Dadurch ist das Risiko psychischer Erkrankungen im Alter erhöht. Diese Menschen sind anfälliger für umgebungsbedingte und soziale Belastungen.

Veränderungen im gesundheitlichen und funktionellen Bereich scheinen bei älteren Menschen mit geistiger Behinderung deutlich früher aufzutreten als bei Personen, die nicht geistig behindert sind. Insbesondere bei Menschen mit DownSyndrom scheint der Alterungsprozess nicht nur früher einzusetzen, sondern sie weisen offensichtlich auch ein höheres Risiko auf, an einer Demenz zu erkranken, und dies bereits in jungen Jahren. Dadurch werden vermutlich viele der alten, vielfach gesundheitlich und funktionell beeinträchtigten Eltern bei der Betreuung von älter werdenden Menschen mit geistiger Behinderung zusätzlich belastet, was möglicherweise einen Umzug der älter werdenden Person mit geistiger Behinderung in eine Einrichtung der Behindertenhilfe notwendig machen kann.

Nimmt man vor dem Hintergrund eines kompetenztheoretischen Konzepts eine Skizzierung der Fähigkeiten von älteren Menschen mit geistiger Behinderung vor, so lassen sich folgende Aussagen treffen: 1. Die Kompetenz im Alter (und zwar sowohl im physischen als auch im seelisch-geistigen Bereich) ist in hohem Maße vom Schweregrad der Behinderung beeinflusst; schon alleine aus diesem Grunde sind Verallgemeinerungen zu vermeiden. Für den Grad der physischen Kompetenz ist weniger die Frage, ob Menschen eine Behinderung aufweisen oder nicht, entscheidend. Vielmehr ist der Grad der Behinderung ein bedeutender Einflussfaktor (Cooper, $1998^{26}$ ). 2. Die Kompetenz im Alter ist in hohem Maße vom Grad der Förderung beeinflusst, die Menschen im Lebenslauf erfahren haben. 3. Die Kompetenz im Alter ist in hohem Maße vom Grad der sensorischen, kognitiven und sozialen Anregungen beeinflusst, die Menschen in früheren Lebensjahren erfahren haben und aktuell erfahren. Dabei ist speziell bei älteren Menschen mit geistiger Behinderung zu berücksichtigen, dass bei diesen Seh- und Höreinbußen häufiger auftreten als bei Menschen ohne Behinderung bzw. mit körperlicher oder seelischer Behinderung (Warburg, 2001 $1^{27}$ ). 4. Der Alternsprozess verläuft bei Menschen mit geistiger Behinderung nicht grundsätzlich anders als bei Menschen ohne geistige Behinde-

25 Maaskant et al. (1996), Care dependence and activities of dailiy living in relation to ageing: results of a longitudinal study, Journal of Intellectual Disability Research, 40, 535-543.

26 Cooper (1998), Clinical study of the effects of age on the physical health of adults with mental retardation, American Journal on Mental Retardation, 102, 582-589.

27 Warburg (2001), Visual impairment in adult people with intellectual disability. Literature review, Journal of Intellectual Disability Research, 45, 424-438. 
rung (Evenhuis, 200128). 5. Die Variabilität im Alter ist bei Menschen mit geistiger Behinderung noch stärker ausgeprägt als bei Menschen ohne geistige Behinderung. 6. Der Kreativität geistig behinderter Menschen ist im Alter genauso wenig eine Grenze gesetzt wie in früheren Lebensaltern: $\mathrm{Zu}$ nennen sind kreative Leistungen im künstlerischen Bereich. 7. Gefühle der Selbstverantwortung und Mitverantwortung sind bei Menschen mit geistiger Behinderung in gleicher Weise vorhanden wie bei Menschen ohne diese Behinderung. Aus diesem Grunde wird der Möglichkeit, auch etwas für andere - zum Beispiel für jüngere Menschen - zu tun, große Bedeutung beigemessen (Hensel et al., 2002 29 ; Meininger, 2000 $0^{30}$ ). 8. Fehlen systematische Anregung oder systematisches Training, so besteht bei Menschen mit geistiger Behinderung die besondere Gefahr, dass die im Lebenslauf entwickelten Fähigkeiten und Fertigkeiten rasch verloren gehen. Aus diesem Grunde ist für Menschen mit geistiger Behinderung das Training wichtig, weil sonst alle früheren Erfolge in Frage gestellt werden (Weltgesundheitsorganisation, 2001 ${ }^{31}$; Zigman et al., 2002 ${ }^{32}$ ). 9. Aufgrund verringerter affektiver und emotionaler Kontrolle sind die Belastungs- und Trauerreaktionen bei Menschen mit geistiger Behinderung intensiver. Aus diesem Grunde muss nach dem Auftreten von Verlusten eher mit tiefgreifenden psychischen Reaktionen gerechnet werden. 10. Die körperliche Ermüdung und seelische Erschöpfung nehmen bei Menschen mit geistiger Behinderung im Alter besonders stark zu, der Antrieb ist verringert. 11. Bei einzelnen Formen geistiger Behinderung - hier ist vor allem das Down-Syndrom zu nennen - ist die Gefahr des Auftretens einer Demenz im Alter erkennbar erhöht. Aus diesem Grunde ist hier dem alltagspraktischen und kognitiven Training besondere Bedeutung beizumessen, damit auch im Verlauf dieser Erkrankung die bestehenden Fähigkeiten und Fertigkeiten möglichst lange erhalten bleiben. 12. Bei der Entwicklung von Förder- und Rehabilitationskonzepten ist darauf zu achten, dass a. an den früheren Förderansätzen angeknüpft und das Training spezifischer Funktionen und Fertigkeiten im Alter fortgesetzt wird, dass b. auch nach Ausscheiden aus der Werkstätte ein ausreichendes Maß an Tagesstrukturierung und sinnerfüllter Aktivität gegeben ist, dass c. Möglichkeiten zur Kreativität bestehen, dass d. Möglichkeiten des körperlichen Trainings (vor allem: Ausdauer, Kraft, Geschicklichkeit) sowie des geistigen und alltagspraktischen Trainings gegeben sind, dass e. bei der Umweltgestaltung die Forderung nach Barrierefreiheit, nach optimalem Anregungsgehalt und nach Hilfsmittels berücksichtigt

Evenhuis (2001), Healthy aging - adults with intellectual disabilities: Physical health issues, Journal of Applied Research in Intellectual Disabilities, 14, 175-194.

29 Hensel et al. (2002), Subjective judgements of quality of life: a comparison study between people with intellectual disability and those without disability, Journal of Intellectual Disabi-lity Research, 46, 95-107.

30 Meininger (2000), Autonomy and professional responsibility in care for persons with intellectual disabilities, Nursing Philosophy, 2, 240-250.

31 World Health Organization (2001), Healthy Ageing - Adults with intellectual disabilities: Summative Report, Journal of Applied Research in Intellectual Disabilities, 14, 256-275.

32 Zigman et al. (2002), Incidence and temporal patterns of adaptive behavior change in adults with mental retardation, American Journal of Mental Retardation, 107, 161-174. 
werden (Jones et al., 1999 $9^{33}$; Kruse et al., 2002 $2^{34}$ ). 13. Es sollte darauf geachtet werden, dass sich Menschen auf das Ausscheiden aus der Werkstätte vorbereiten können und der Übergang in die nachberufliche Zeit gleitend erfolgt. Kontakte zur Werkstätte sollten auch nach Ausscheiden aus dem Beruf prinzipiell bestehen bleiben, wenn der Wunsch danach besteht. Ältere Menschen mit geistiger Behinderung sind gerade in der Phase des Übergangs in die nachberufliche Zeit in hohem Maße verunsichert, inwieweit sie auch in Zukunft Kontakte finden und persönlich bedeutsamen Tätigkeiten nachgehen können. Weiterhin gewinnt die Fragen der sozialen Anerkennung nach Ausscheiden aus dem Beruf Bedeutung (Gusset-Bährer, 2003 ${ }^{35}$ ). 14. Aufgrund abnehmender körperlicher Belastungsfähigkeit oder des Todes der Eltern ist die familiäre Betreuung der Menschen mit geistiger Behinderung nicht gesichert oder sogar unmöglich. Alternative Formen des gemeinschaftlichen Wohnens müssen geschaffen werden. Dabei ist in besonderem Maße auf die Erhaltung der sozialen Integration und Partizipation der Menschen mit geistiger Behinderung zu achten. Diese müssen die Möglichkeit haben, in einem ihnen vertrauten Umfeld mit ihnen vertrauten Menschen zu leben. Aus diesem Grunde ist im Falle von Pflegebedarf auch das Verbleiben in der Behinderteneinrichtung dem Umzug in ein Altenpflegeheim vorzuziehen. Der Umzug wäre vielfach mit einer Aufgabe des vertrauten sozialen Umfelds verbunden, die gerade von Menschen mit geistiger Behinderung nur schwer verarbeitet werden kann. Abgesehen davon ist in den meisten Altenpflegeheimen die für die Betreuung behinderter Menschen notwendige heilpädagogische Expertise nicht vorhanden. Einrichtungen der Behindertenhilfe stehen vor der Aufgabe, Expertise im Bereich der Altenpflege zu erwerben (sei es durch die Einstellung von Altenpflegern, sei es durch die Weiterbildung der Heilerziehungspfleger im Bereich der Altenpflege). Gegebenenfalls können in den Einrichtungen der Behindertenhilfe eigene Pflegeabteilungen geschaffen werden. Entscheidend ist die Integration von Elementen der Behinderten- und Altenhilfe - ein Aspekt, auf den später noch ausführlicher einzugehen sein wird.

\section{Behinderungen als Folge spezifischer Person-Umwelt-Interaktionen}

Die hier vorgeschlagene Definition von Kompetenz rückt sowohl die Person als auch die Umwelt in das Zentrum. Sie konzentriert sich zum einen auf die Fähigkeiten der Person, zum anderen betrachtet sie diese im Kontext der jeweils gegebenen räumlichen, sozialen und infrastrukturellen Umwelt: Inwieweit fördert, inwieweit

33 Jones et al (1999), Opportunity and promotion of activity among adults with severe intellectual disability living in community residences: the impact of training staff in active support, Journal of Intellectual Disability Research, 43, 164-178.

34 Kruse et al. (2002), Den Jahren Leben geben. Lebensqualität im Alter bei Menschen mit Behinderungen, Projektbericht, Stuttgart: Diakonisches Werk Württemberg.

35 Gusset-Bährer (2003), Menschen mit geistiger Behinderung beim Übergang vom Beruf in die nachberufliche Phase, Phil. Diss. Universität Heidelberg: Fakultät für Empirische Kultur- und Sozialwissenschaften. 
erschwert diese die Erhaltung oder Wiederherstellung eines selbstständigen, selbstverantwortlichen und sinnerfüllten Lebens? Mit der Akzentuierung der Wechselwirkungen zwischen Person und Umwelt wird verdeutlicht, dass Behinderungen nicht allein als Merkmal der Person zu verstehen sind, dass also die Umwelt Mitverantwortung für die Kompetenz des Menschen trägt.

Hingegen werden Behinderungen in der öffentlichen Diskussion primär oder ausschließlich aus einer personzentrierten, medizinisch-kurativen Perspektive betrachtet. Behinderung bildet danach allein ein Merkmal der Person; der Medizin, der Rehabilitation, der Heilpädagogik wird die Aufgabe gestellt, zur Linderung oder Kompensation bestehender Funktions- und Fähigkeitseinbußen beizutragen. Diese Perspektive verdeckt jedoch die Bedeutung der räumlichen, sozialen und infrastrukturellen Umwelt für die Behinderung. Behinderung ist auch Folge von behindernden Umweltbedingungen. Die nachfolgend angeführte Definition von Behinderung macht deutlich, dass eingeschränkte Funktionen und Fertigkeiten vor allem im umweltbezogenen Handeln des Menschen offenbar werden:

„Behinderung ist nicht in erster Linie ein Synonym für eine medizinische Diagnose, sondern ein umfassendes personales und soziales Geschehen. Behinderung stellt sich dar als ein auf mehreren Wirkungsebenen laufender Prozess. Diese Ebenen bezeichnen den unmittelbar aus dem klinischen Krankheitsgeschehen resultierenden Schaden (Impairment), die individuellen und funktionellen Einschränkungen mit der Folge von unterschiedlichen Fähigkeitsstörungen (Disability) und die soziale Beeinträchtigung (Handicap) und die sich daraus ergebenden vielfältigen persönlichen, familiären und gesellschaftlichen Folgen“ (Paeslack, 1998 ${ }^{36}$ ).

Für Heilpädagogik und Rehabilitation ergeben sich aus dieser Definition von Behinderung die folgenden fünf Aufgaben: 1. Umweltbedingungen sind so zu verändern, dass Menschen trotz bestehender Einbußen spezifischer Fähigkeiten in der Lage sind, in ihrer vertrauten (räumlichen und sozialen) Umwelt selbstständig und selbstverantwortlich zu leben sowie sozial zu partizipieren. 2. Barrieren in der räumlichen Umwelt, die sich negativ auf Mobilität und Selbstständigkeit auswirken, sind zu beseitigen. 3. (Technische) Hilfsmittel, die Mobilität, Selbstständigkeit und Kommunikationsfähigkeit fördern, sind in die räumliche Umwelt zu integrieren. 4. Beim Erwerb von Kenntnissen und Fertigkeiten zum effektiven Umgang mit diesen Hilfsmitteln ist Unterstützung anzubieten. 5. In unserer Gesellschaft ist auf ein verändertes Verständnis von Behinderung hinzuwirken, das a. Behinderung nicht mit Krankheit gleichsetzt, b. die Verschiedenartigkeit der Lebensbedingungen, Bedürfnisse und Fähigkeiten von Menschen mit Behinderung wahrnimmt und damit die Notwendigkeit eines differenzierten Spektrums an Angeboten (Tagesgestaltung, Wohnen, Bildung, Aktivierung) anerkennt, c. die große Bedeutung der räumlichen, sozialen und infrastrukturellen Umwelt für die Folgen von Behinderung akzentuiert. Die genannten Aufgaben der Rehabilitation lassen sich auch in der Weise charakterisieren, dass Abhängigkeit und Fremddefinition in den Lebensbereichen Wohnen, Arbeit und Freizeit zugunsten einer selbständigen und selbstverantwortlichen Le- 
bensgestaltung aufgehoben werden. Diese umfassende Zielsetzung liegt auch den Leitbegriffen Normalisierung und Integration zugrunde.

Vorbilder für dieses veränderte Verständnis von Behinderung finden sich unter anderem in Großbritannien. Dort nimmt die Umweltgestaltung (zu der auch die Ausstattung mit mobilitäts-, selbstständigkeits- und kommunikationsfördernden Hilfsmitteln gehört) bei der Entwicklung von Förderkonzepten sowie im Prozess der Betreuung und Rehabilitation eine hervorgehobene Stellung ein. Als Beispiel sei eine Erklärung der British Psychological Society aus dem Jahre 1989 angeführt, in der als Aufgabe des Sozial- und Gesundheitswesens vor allem die Linderung sozialer Handicaps genannt wird:

\begin{abstract}
„Die Konzentration allein auf den behinderten Menschen geht an der Tatsache vorbei, dass die Gesellschaft in hohem Maße körperlich unversehrte Menschen bevorzugt, hingegen Menschen mit Fähigkeitsstörungen ausschließt; dies ist zum Beispiel in der Arbeitwelt der Fall, dies zeigt sich aber auch in der baulichen Gestaltung öffentlicher Gebäude. Es ist notwendig, soziale Handicaps zu verändern, anstatt Menschen mit Behinderung zu zwingen, sich willkürlich aufgestellten Normen anzupassen. Diese Normen werden in aller Regel von Menschen entwickelt, die körperlich unversehrt sind“".
\end{abstract}

Diese Aussage ist repräsentativ für jene Einrichtungen in Großbritannien, die an der Entwicklung von Förder-, Betreuungs- und Rehabilitationskonzepten für Menschen mit Behinderung beteiligt sind. Die dort entwickelten Modelle von Behinderung rücken die sozialen Barrieren in den Vordergrund. Aus diesen Modellen ergeben sich folgende Veränderungen in den bereitgestellten Hilfen: 1. Diese sollen sich nicht allein auf Gesundheit und Versorgung beschränken, sondern in gleichem Umfang Veränderungen in der Umwelt einschließen (erhöhter Anregungsgehalt, Zugänglichkeit von öffentlichen Räumen und sozialen Dienstleistungen, Stärkung der Kontrolle über die Umwelt, Stärkung der sozialen und kulturellen Teilhabe). 2. Hilfen für behinderte Menschen sollen nicht länger im Sinne verordneter und erbrachter Dienste verstanden werden, sondern im Sinne umweltbezogener Ressourcen, auf die behinderte Menschen einen gesetzlich geregelten Anspruch haben. 3. Hilfen für behinderte Menschen sollen in Richtung auf ein integriertes Unterstützungssystem gestaltet werden. Die von behinderten Menschen genutzten Hilfen - wie medizinische Dienste, Bildungsangebote, Gestaltung des Wohnraums, Transporthilfen müssen stärker aufeinander abgestimmt sein, sie sind als modulares System und nicht als unabhängig voneinander bestehende Komponenten zu verstehen. 4. Ausbildung und Training von Dienstleistenden im Bereich der (technischen) Hilfen sollen so beschaffen sein, dass deren Fertigkeiten noch stärker auf das Erkennen und die Beseitigung von Barrieren gerichtet sind. 5. Die gesetzlichen Bedingungen müssen in der Hinsicht weiterentwickelt werden, dass Menschen mit Behinderungen in ihrer eigenen Wohnung leben können und gleiche Möglichkeiten zur Nutzung von medizinischen Angeboten sowie von Bildungs- und Freizeitangeboten haben; darüber hinaus ist ihren Bedürfnissen bei der Gestaltung der Wohnung und des Wohnumfeldes Rechnung zu tragen. 


\section{Die infrastrukturelle Umwelt: Inwieweit spiegelt sich die Kompetenzorientierung in den spezifischen Angeboten wider?}

In einer Studie von Moss $\left(1992^{37}\right)$ wurden neun Länder in Bezug auf die spezifischen Formen der Behindertenhilfe miteinander verglichen. Vor allem in den Niederlanden und in Deutschland wurde die institutionelle Betreuung und Pflege als zentrale Komponente innerhalb des Spektrums von Dienstleistungen interpretiert. In Nordamerika, Australien und Großbritannien hingegen wurde deutlich größeres Gewicht auf den Abbau der Institutionalisierung und die Integration in der Kommune gelegt. - Allerdings, so hebt Moss (1992) hervor, ist die Verfügbarkeit von Plätzen außerhalb der Einrichtungen in den 80er Jahren des vergangenen Jahrhunderts nur in geringem Umfang gestiegen. Wenn Familien wünschen, dass Angehörige außerhalb von Einrichtungen leben, so müssen sie damit rechnen, auf eine lange Warteliste zu kommen. Moss (1992) deutete das Bedürfnis nach einem Platz außerhalb einer Einrichtung der Behindertenhilfe in den neun Ländern als das wichtigste der nicht verwirklichten Bedürfnisse. Für die Bundesrepublik Deutschland gilt auch heute noch die Aussage, dass Angebote der Behindertenhilfe primär institutioneller Art sind und eine „De-Institutionalisierung“ noch nicht wirklich eingeleitet wurde. Diese Aussage ist nicht als ein Vorwurf an Institutionen zu verstehen. Es wird hier auch nicht die Annahme vertreten, dass außerhalb von Institutionen grundsätzlich die besseren Angebote erfolgten und zudem der Individualisierungsgrundsatz eher zu verwirklichen sei. Vielmehr ist der positive Effekt einer zunehmenden DeInstitutonalisierung darin zu sehen, dass sich mehr Wohnalternativen ausbilden können. Diese sind angesichts der Tatsache, dass Menschen mit Behinderung eine heterogene Gruppe bilden, sinnvoll und können - im Sinne der anregenden und unterstützenden Umwelt - dazu beitragen, dass Selbstständigkeit, Selbstverantwortung und Sinnerfahrung erhalten bleiben oder gefördert werden (Moss, 1994 ${ }^{38}$ ).

Die Forderung, vermehrt Angebote außerhalb von Institutionen zu schaffen, kann nur dann erhoben werden, wenn auch in der Kommune Möglichkeiten bestehen, ältere Menschen mit Behinderung bei der Verwirklichung des Bedürfnisses nach sozialer Integration und Teilhabe zu unterstützen - ohne ihnen allerdings die Selbstverantwortung bei der Gestaltung des Alltags und der sozialen Beziehungen zu nehmen. In den Vereinigten Staaten sind mehrere Modellprojekte durchgeführt und evaluiert wurden, die dazu dienten, Möglichkeiten der Förderung von sozialer Integration und Teilhabe außerhalb von Institutionen zu prüfen.

In einem US-amerikanischen Modell mit dem Titel 'Senior Center - Senior Companion' (Heller, 1999 ${ }^{39}$ ) wurde das Ziel verfolgt, die Integration älterer Men-

Moss (1992), Aging and developmental disabilities: Perspectives from nine countries, Durham: World Rehabilitation Fund \& University of New Hamsphire.

38 Moss (1994), Quality of life and aging, in: Goode (Ed.), Quality of life for persons with disabilities (pp. 218-234), Cambridge: Brookline Books.

39 Heller (1999), Emerging models, in: Herr/Weber (Eds.), Aging, right, and quality of life (pp.149166), Baltimore: Brookes. 
schen mit Behinderung in der Gemeinde durch Unterstützung von Mitarbeitern, ehrenamtlich Tätigen und Freunden zu erreichen. Die unterstützenden Personen wurden dabei als Mentoren angesehen, die ältere Menschen mit Behinderung in soziokulturelle Einrichtungen für ältere Menschen ohne Behinderung einführen sowie in andere kommunale Aktivitäten einbinden sollten. Dadurch sollten soziale Bezugssysteme geschaffen werden, die zur sozialen Integration und sozialen Teilhabe beitragen. Die Evaluation dieses Modell ergab Erfolge im Hinblick auf eine Steigerung der Lebensqualität durch 1. die vermehrte (objektive und subjektiv erlebte) soziale Integration, 2. das erweiterte Spektrum an Aktivitäten im Alltag, 3. das verbesserte Selbstkonzept.

Factor und Anderson $\left(1992^{40}\right)$ haben in einer für die Vereinigten Staaten repräsentativen Studie untersucht, welche innovativen Programme und Maßnahmen zur Individualisierung und sozialen Teilhabe älterer Menschen mit geistiger Behinderung entwickelt wurden. Die von ihnen als innovativ eingeschätzten Programme und Maßnahmen zeichneten sich vor allem durch folgende sechs Merkmale aus:

- Individuelle Trainingsangebote zur Förderung des Entscheidungsverhaltens

- Vermittlung von Informationen über Dienstleistungen in der Kommune

- Unterstützung bei der sozialen und kulturellen Teilhabe in der Kommune

- Bereitstellung von technischen Hilfen und Unterstützung bei deren Nutzung

- Vermehrte Individualisierung in der Arbeitsplatz- und Arbeitszeitgestaltung

- Einbeziehung von ehrenamtlich tätigen Menschen in die Arbeit mit behinderten Menschen

Im Kontext der Bereitstellung und Unterstützung bei der Nutzung von Technik ist folgender Befund von Bedeutung: Trotz der Fortschritte bei der Entwicklung von nutzerfreundlicher Technik wird diese speziell von älteren Menschen mit geistiger Behinderung nicht genutzt. Bereits zu Beginn der 90er Jahre wurde eine Untersuchung veröffentlicht, aus der hervorgeht, dass speziell jene älteren Menschen mit geistiger Behinderung, die außerhalb von Einrichtungen leben, nicht über technische Hilfsmittel verfügen, durch die deren Selbstständigkeit, Selbstverantwortung und soziale Integration gefördert werden könnte. In dieser Gruppe wurden durchschnittlich zwei nicht erfüllte Bedürfnisse nach technischen Hilfen pro Person ermittelt (Mann et al., $1993^{41}$ ).

Frage nachgegangen, durch welche Maßnahmen dazu beigetragen werden kann, dass Menschen mit Behinderung vermehrt am Leben in der Gemeinde teilhaben. Es wurden von den befragten Experten die folgenden sieben Maßnahmen als zentral erachtet:

1. Die Familie und die Gemeinde müssen als Ressourcen verstanden werden, die stärker genutzt werden sollten

40 Factor/Anderson (1992), Person-centered planning innovative approaches in case management and habilitation planning, New Orleans: American Association on Mental Retardation.

41 Mann et al. (1993), Needs of home-based older persons for assistive devices, Technology and Disability, 2, 1-11. 
2. Die zwischenmenschlichen Beziehungen, vor allem die Freundschaften, müssen eine deutlich größere Beachtung finden

3. Bei der Konzeptentwicklung sind die individuellen Lebenspläne sehr viel stärker zu berücksichtigen

4. Individuelle Entscheidungsprozesse müssen gefördert werden - es sollen eben nicht Entscheidungen abgenommen werden, sondern vielmehr soll zur selbstverantwortlichen Entscheidung motiviert werden. Wenn Unterstützung im Prozess der Entscheidung notwendig ist, so darf diese keinesfalls die individuellen Entscheidungsspielräume einschränken

5. Menschen müssen dabei unterstützt werden, Kontrolle über ihre Umwelt auszuüben

6. Die Assistenz in der vertrauten Umgebung sowie die unterstützende Technologie müssen sehr viel stärker beachtet werden

7. Es ist größeres Gewicht auf die Lebensqualität sowie auf die individuellen Kriterien für Lebensqualität zu legen

Zahlreiche Projekte zentrieren sich um die Förderung individueller Entscheidungskompetenz durch spezifische Bildungsansätze. Dabei kann immer wieder beobachtet werden, dass schon durch geringfügige Interventionen mit dem Ziel, die Entscheidungskompetenz des Individuums zu erhöhen (zum Beispiel in Bezug auf die Ausführung bestimmter Aktivitäten des täglichen Lebens oder die Gestaltung des Alltags), auch bei Menschen mit Behinderung eine Zunahme der Motivation, eigene Entscheidungen zu treffen, erreicht werden kann. Die Fähigkeit, Entscheidungen zu treffen, wird dabei als zentral für die Lebensqualität des Menschen betrachtet. Denn die Fähigkeit, Entscheidungen zu treffen, ist nicht nur Ausdruck von Autonomie und Kompetenz, sondern sie stellt zudem eine bedeutsame Maßnahme zur Erfüllung persönlicher Ziele dar.

Die Förderung der Entscheidungskompetenz dient auch der Stärkung persönlicher Kontrolle des Individuums über Ereignisse in seiner sozialen Umwelt (Beispiele: Einfluss auf das Verhalten anderer Menschen nehmen, das Verhalten anderer Menschen besser vorhersagen können, Gewissheit, dass eigene Werte und Bedürfnisse von anderen Menschen respektiert werden). Der Verlust persönlicher Kontrolle ist vielfach für die Entstehung von Depressionen verantwortlich. Zudem wird „schwieriges“ oder „herausforderndes“ Verhalten von Menschen, die nur über geringe Kontrolle verfügen, als Möglichkeit wahrgenommen, trotzdem Kontrolle über ihre Umwelt auszuüben.

Sowohl im Forschungsteam von Heller als auch im Forschungsteam von Haveman wurde Lernmaterial zur Förderung von Entscheidungskompetenz entwickelt und evaluiert. Dieses Material wird als Teil eines umfassenden Bildungskonzepts verstanden. Im Zentrum der von Heller und Mitarbeitern entwickelten Lerneinheit steht die Vermittlung von Wissen und Fertigkeiten in folgenden Bereichen: 1. Der Prozess der Entscheidungsfindung: Wie können individuell und sozial verantwortliche Entscheidungen getroffen werden? (Hier findet sich eine Übereinstimmung mit den beiden zentralen Kategorien der Selbstverantwortung und der Mitverantwor- 
tung.) 2. Die Rechte und Pflichten des Individuums. 3. Selbstbestimmung bei der Nutzung der freien Zeit sowie bei der Gestaltung des Alters. 4. Gesundheitsbewusste Lebensführung. - Die von Haveman ausgearbeitete Lerneinheit zum Thema „Selbstbestimmt älter werden" setzt sich aus folgenden sieben Lernschritten zusammen: 1. Selbstbestimmt Entscheidungen treffen. 2. Den Übergang in den Ruhestand vorbereiten (hier wird auch die Einbeziehung flexibler Arbeitszeitmodelle empfohlen). 3. Wohnformen kennen lernen. 4. Gründung und Pflege sozialer Beziehungen. 5. Erwerb von Kompetenzen für eine selbstverantwortliche Freizeitgestaltung. 6. Ausbildung der Bereitschaft und der Fertigkeiten zur gesundheitsbewussten Lebensführung. 7. Fähigkeit zur psychischen Auseinandersetzung mit Erkrankungen.

Ein spezielles Programm von Heller zielte auf die Vorbereitung von älteren Menschen mit geistiger Behinderung auf den Austritt aus dem Berufsleben (Heller et al., $1996^{42}$ ). Dieses Programm, welches unter der Überschrift „Person zentrierte Lebensplanung für das höhere Lebensalter " stand, umfasste folgende Bildungseinheiten:

- Training in Entscheidungsverhalten

- Training in Zieldefinition

- Informationen über Möglichkeiten, Gesundheit und Wohlbefinden zu erhalten

- Informationen über Freizeitaktivitäten und freiwillige Tätigkeiten im Ruhestand

- Informationen über Wohnformen und Freundschaften

- Training für Mitarbeiter von Einrichtungen und Familienangehörige mit dem Ziel, diese in die Lage zu versetzen, ältere Menschen mit geistiger Behinderung bei der Lebensplanung zu unterstützen

Die genannten Projekte sind Beispiele für die seit Beginn der 90er Jahre im internationalen und nationalen Raum zu beobachtende Akzentsetzung im Hinblick auf die Förderung von Selbstverantwortung. Die Hauptanliegen dieser Projekte lassen sich dabei in zwei Punkten zusammenfassen: Zum einen soll die Fähigkeit zum selbstständigen und selbstverantwortlichen Leben erhalten oder gefördert werden, zum anderen soll der Umzug in eine Pflegeeinrichtung aufgrund eingetretenen Hilfeoder Pflegebedarfs vermieden werden. Die Ergebnisse entsprechender Projekte (siehe auch Kruse et al., 2002 ${ }^{43}$ ) stützen die Annahme der auch bei älteren Menschen mit geistiger Behinderung gegebenen Lernfähigkeit. Schon ein drei bis vier Monate dauerndes Training in Selbstsicherheit und Entscheidungsverhalten führt zu statistisch signifikanten Verbesserungen in diesen beiden Merkmalen. Aus diesem Grunde ist es gerechtfertigt, die Förderprogramme für ältere Menschen mit geistiger Behinderung auch aus einer Bildungsperspektive zu betrachten (zum Beispiel Kruse,

42 Heller et al. (1996), Impact of person-centered later life planning training program for older adults with mental retardation, Journal of Rehabilitation, 62, 77-83.

43 Kruse et al. (2002), Den Jahren Leben geben. Lebensqualität im Alter bei Menschen mit Behinderungen, Projektbericht, Stuttgart: Diakonisches Werk Württemberg. 
$1997^{44}$; Kruse et al., 2002 $2^{45}$ ), die ihrerseits ein zentrales Merkmal der Eingliederungshilfe darstellt.

Die Ergebnisse deuten zudem auf die auch bei älteren Menschen mit geistiger Behinderung gegebene kognitive Plastizität und Verhaltensplastizität. Im allgemeinen Sinne beschreibt Plastizität die Reservekapazität der Nervenzelle, das heißt, das Potenzial zu erhöhter Reaktions- und Anpassungsfähigkeit, welches unter Aktivierung ausgeschöpft wird. Bei fehlender Aktivierung wird hingegen dieses Potenzial nicht genutzt; die Zelle zeigt nicht jene Reaktions- und Anpassungsfähigkeit, die sie potenziell erbringen könnte. Die Verhaltens- und kognitive Plastizität im hohen Lebensalter konnte zum Beispiel in Studien nachgewiesen werden, in denen potenzielle Wirkungen einer selbstständigkeitsfördernden Umwelt auf die alltagspraktische Kompetenz untersucht wurden (siehe dazu M. Baltes, 1995 ${ }^{46}$; Zank \& Baltes, $1998^{47}$ ). In diesen Studien wurde zunächst aufgezeigt, dass das Verhalten von Pflegefachkräften als ,,selbstständigkeitsfördernd“ vs. „,abhängigkeitsfördernd“ klassifiziert werden kann: Im ersten Falle (selbstständigkeitsfördernd) unterstützen Pflegefachkräfte die Bewohner in deren Bemühen, Aktivitäten des täglichen Lebens möglichst selbstständig auszuführen - sie geben diesen mehr Zeit für die Ausführung dieser Aktivitäten, sie beraten diese, sie greifen möglicht selten in die Ausführung einer Handlung ein, sie verstärken selbstständigkeitsorientiertes Verhalten und ignorieren abhängigkeitsorientiertes Verhalten der Bewohner. Im letzteren Falle (abhängigkeitsfördernd) behindern Pflegefachkräfte die Bewohner in deren Bemühen, Aktivitäten des täglichen Lebens selbstständig auszuführen - indem sie in die Ausführung von Handlungen direkt eingreifen, stellvertretend für den Bewohner Handlungen übernehmen, selbstständigkeitsorientiertes Verhalten ignorieren und abhängigkeitsorientiertes Verhalten verstärken. Je nach Orientierung der Pflegefachkräfte zeigen die Bewohner unterschiedliche Grade von Selbstständigkeit: Im ersten Falle ist eine deutliche Zunahme der Selbstständigkeit, im letzteren Falle eine deutliche Zunahme der Abhängigkeit erkennbar. Selbstständigkeitsfördernde Umwelten können auch im Sinne der Aktivierung und Nutzung von Reservekapazitäten verstanden werden, abhängigkeitsfördernde Umwelten hingegen im Sinne von Kontexten, welche die Reservekapazität (Plastizität, Veränderungspotenzial) des Zentralnervensystems ungenutzt lassen. Das im Arbeitskreis von M. Baltes entwickelte Forschungsparadigma wurde von Kruse et al. $\left(2002^{48}\right)$ auf die Analyse der Konzepte in der

44 Kruse (1997), Bildung und Bildungsmotivation im Erwachsenenalter, in: Weinert/Mandl (Hrsg.), Enzyklopädie der Psychologie - Pädagogische Psychologie: Psychologie in Erwachsenenbildung (S. 120-166), Göttingen: Hogrefe.

45 Kruse et al. (2002), Medizinpsychologische und -soziologische Aspekte des Alterns, in: Brähler/Strauß (Hrsg.), Handlungsfelder in der Psychosozialen Medizin (S. 163-184), Göttingen: Hogrefe.

46 Baltes (1995), Verlust der Selbstständigkeit im Alter: Theoretische Überlegungen und empirische Befunde, Psychologische Rundschau, 46, 159-170.

47 Zank/Baltes (1998), Förderung von Selbständigkeit und Lebenszufriedenheit alter Menschen in stationären Einrichtungen, in: Kruse (Hrsg.), Psychosoziale Gerontologie, Band 2: Intervention (S. 60-72), Göttingen: Hogrefe.

Kruse et al. (2002), Den Jahren Leben geben, Stuttgart: Verlag Diakonisches Werk. 
Behindertenhilfe übertragen. Auch hier wurde eine Differenzierung nach „selbstständigkeits- vs. abhängigkeitsfördernder“ Betreuung und Pflege vorgenommen; es wurden - ganz ähnlich wie im Arbeitskreis von M. Baltes - Interventionen bei Mitarbeitern durchgeführt, die dazu dienen sollten, deren Selbstständigkeitsorientierung im Betreuungs- und Pflegeverhalten zu fördern. Es ließ sich zeigen, dass selbstständigkeitsorientiertes Verhalten der Mitarbeiterinnen und Mitarbeiter mit einer signifikanten Zunahme der Selbstständigkeit, Selbstverantwortung und subjektiv erlebten Kompetenz bei den Bewohnern einhergeht. Diese Untersuchungsbefunde sprechen nicht nur für die positiven Effekte der selbstständigkeitsorientierten Betreuung und Pflege. Sie deuten auch auf die bei älteren Menschen mit geistiger Behinderung bestehende Verhaltens- und kognitive Plastizität. - In dieser Studie wurde an die Mitarbeiterinnen und Mitarbeiter abschließend folgende Frage gerichtet: „Was können Sie in Ihrer täglichen Arbeit tun, um die Kompetenz der älter werdenden und alten Menschen mit geistiger Behinderung möglichst lange zu erhalten und zu fördern"? Nachfolgend sind die zentralen Kategorien aufgeführt, um die sich die Antworten zentrierten und die somit den Kern der Betreuungs- und Pflegekonzepte bilden:

1. Sicherheit bieten

- durch akzeptierende Grundhaltung

- durch feste Bezugspersonen

- durch gleiche Abläufe

2. Erhaltung des Aufgabenspektrums in der Wohngruppe

3. Aktivierung

- durch körperliche Mobilisierung

- durch anregende Umweltgestaltung

4. Unterstützung selbstständigkeitsorientierten Verhaltens

5. Förderung sozialer Kontakte

6. Individuelle Betreuungsplanung, Betreuung und Förderung

7. Anpassung an die Ressourcen der Person

- mehr Zeit geben

- die Anforderungen den Fähigkeiten anpassen

8. Ermöglichen von Erfolgserlebnissen

9. Entgegenbringen von Respekt

Vor dem Hintergrund der hier kurz skizzierten Modelle und Untersuchungsergebnisse lassen sich die zentralen Anforderungen an kompetenzfördernde Konzepte in der Behindertenhilfe wie folgt zusammenfassen: 1. Es ist an früheren Förderansätzen anzuknüpfen. 2. Die Nutzung und das Training spezifischer Funktionen und Fertigkeiten sind fortzusetzen. 3. Nach Ausscheiden aus der Werkstätte für Behinderte ist ein ausreichendes Maß an Tagesstrukturierung sicherstellen. 4. Es sind Möglichkeiten zur Kreativität, zum körperlichen Training (Ausdauer, Kraft, Geschicklichkeit) und zum alltagspraktischen Training zu schaffen. 5. Nach Ausscheiden aus der Werkstätte für Behinderte sollten die Kontakte zu dieser bestehen bleiben. 6. Bei der Entwicklung kompetenzfördernder Konzepte ist von lebenslanger 
Lern- und Entwicklungsfähigkeit auszugehen. Im Rahmen eines Gesamtplans (§ 46 BSHG) ist das angemessene individuelle Hilfeangebot fortzuschreiben. 7. Dem Wunsch- und Wahlrecht von behinderten Menschen kommt im Alter ganz besondere Bedeutung zu. Hier ist die Flexibilisierung der Angebote wichtig, auch erhält die Beratung großes Gewicht. 8. Menschen mit Behinderung sollen in der Einrichtung bzw. in dem ihn bekannten Wohnumfeld verbleiben können, um somit die Kontinuität des Lebensumfeldes wahren zu können. In Wohneinrichtungen der Eingliederungshilfe ist das Wohnen in altersheterogenen und altershomogenen Gruppen vorzusehen. 9. Für Menschen mit Behinderung ist es normal, mit spezifischen Hilfen, nämlich mit jenen der Eingliederungshilfe, ein möglichst selbstständiges Leben zu führen. An diesem Hilfebedarf ändert sich prinzipiell nichts bei Erreichen einer definierten Altersgrenze. Insoweit spricht das Normalitätsprinzip für die Beibehaltung der angestammten Lebenszusammenhänge auch im Alter, insbesondere aus sozialen Gründen. 10. Menschen mit Behinderung, die bereits in einer Einrichtung der Eingliederungshilfe gelebt haben, können für sich nach dem Normalitätsprinzip in Anspruch nehmen, dass die Kontinuität ihrer Lebenszusammenhänge gewahrt bleibt, wenn sie einen altersspezifischen Hilfebedarf entwickeln.

Das Pflegeheim ist für Menschen mit Behinderung in der Regel nicht die geeignete Wohn- und Betreuungsform. Ein Umzug nur aufgrund des Ausscheidens aus der Werkstätte für Behinderte oder des Erreichens des 65. Lebensjahres in Pflegeheim darf nicht erfolgen. Um einen Umzug in eine Pflegeeinrichtung zu vermeiden, sind in Einrichtungen der Behindertenhilfe Pflegeangebote zu schaffen. Es bedarf hier der entsprechenden räumlichen Gestaltung und sächlichen Ausstattung in den Wohneinrichtungen. Die Empfehlung, dass ältere Menschen mit Behinderung in ihrem gewohnten Lebensumfeld verbleiben sollten und aus diesem Grunde der Umzug in eine Einrichtung der Altenhilfe möglichst zu vermeiden sei, ist nur dann zu verwirklichen, wenn Mitarbeiter in Einrichtungen der Behindertenhilfe über ausreichende Kompetenzen in der Altenhilfe verfügen - zu nennen sind hier Grundlagenkenntnisse in Fragen der Gerontologie, der Geriatrie, der Gerontopsychiatrie, der aktivierenden Pflege. Umgekehrt müssen Mitarbeiter jener stationären Einrichtungen der Altenhilfe, die Menschen mit Behinderung aufnehmen, über ausreichende Kompetenzen in der Heilpädagogik (und zwar in beiden Bereichen: Bildung und Rehabilitation) verfügen.

\section{In welcher Hinsicht lassen sich die Kompetenz und Lebensqualität durch die Entwicklung innovativer Konzepte fördern?}

Zunächst werden Aussagen zu zentralen Merkmalen der Kompetenz und Lebensqualität bei Behinderung im höheren Lebensalter getroffen; auch hier steht die Lebenssituation von Menschen mit geistiger Behinderung im Vordergrund. Dabei werden Kompetenz und Lebensqualität noch stärker als bisher aus der Sicht der Intervention betrachtet. - In einem zweiten Schritt werden zentrale Kategorien innovativer Konzepte der Arbeit mit älteren Menschen, bei denen eine Behinderung besteht, disku- 
tiert. Die Diskussion orientiert sich allem an den ,Fachlichen Leitlinien und Empfehlungen', die vom Hessisches Sozialministerium und dem Landeswohlfahrtsverband Hessen in Kooperation mit Wissenschaft und Praxis entwickelt wurden (Hessisches Sozialministerium \& Landeswohlfahrtsverband, $2000^{49}$ ). - In einem dritten Schritt wird die Entwicklung innovativer Konzepte aus der Perspektive der Einrichtungen der Behindertenhilfe sowie der Mitarbeiterinnen und Mitarbeiter dieser Einrichtungen untersucht: In welchen Bereichen der Arbeit mit älteren Menschen, bei denen eine geistige, eine körperliche oder eine seelische Behinderung vorliegt, sehen Mitarbeiterinnen und Mitarbeitern der Behindertenhilfe in Deutschland besonderen Handlungsbedarf? Bei der Beantwortung dieser Frage stützen wir uns auf eine in 45 Einrichtungen der Behindertenhilfe durchgeführte Expertenbefragung.

\subsection{Zentrale Merkmale der Kompetenz und Lebensqualität bei Behinderung im Alter aus einer Interventionsperspektive}

Den Ausgangspunkt jener Arbeiten, in denen der Versuch unternommen wird, Merkmale der Kompetenz und der Lebensqualität zu differenzieren, orientieren sich vielfach an Arbeiten zum ,erfolgreichen Altern“. In einer von P. Baltes \& M. Baltes $\left(1990^{50}\right)$ herausgegebenen Monographie zum „erfolgreichen Altern“ werden sieben Merkmale genannt, die bei einer Definition des Konstrukts zu berücksichtigen sind.

1. Lebensdauer

2. Positiver psychischer Status

3. Gute allgemeine Gesundheit

4. Erhaltene kognitive Kompetenz

5. Kontrolle über das eigene Leben und über die zu verfolgenden Ziele

6. Erhaltene soziale Kompetenz und fortdauerndes produktives Engagement

7. Hohe subjektive Zufriedenheit mit dem eigenen Leben

Es liegen Arbeiten vor, welche die hier genannten Merkmale erfolgreichen Alterns mit Merkmalen der Kompetenz und Lebensqualität von Menschen mit Behinderung verbinden (Hawkins, 1999 $9^{51}$; Schalock, 1996 ${ }^{52}$, 199733; Schalock et al., $1999^{54}$; Taylor \& Bogdan, $1996^{55}$ ). In diesen Arbeiten werden vor allem folgende

49 Hessisches Sozialministerium \& Landeswohlfahrtsverband Hessen (2000), Lebensräume älterer Menschen mit Behinderung. Fachliche Leitlinien und Empfehlungen, Wiesbaden: Hessisches Sozialministerium.

50 Baltes/Baltes (Ed.) (1990), Successful aging: Perspectives from the behavioral sciences, New York: Cambridge University Press.

51 Hawkins (1999), Rights, Place of Residence, and Retirement: Lessons from Case Studies on Aging, in: Herr/Weber (Eds.), Aging, rights, and quality of life (pp. 93-108), Baltimore: Brookes.

52 Schalock (Ed.) (1996), Quality of Life, Vol. I: Conceptualization and measurement, Washington, DC: American Association of Mental Retardation.

53 Schalock (Ed.) (1997), Quality of Life, Vol. II: Application to persons with disabilities, Washington, DC: American Association of Mental Retardation.

54 Schalock et al. (1999), Enhancing quality of life, in: Herr/Weber (Eds.), Aging, rights, and quality of life (pp. 81-92), Baltimore: Brookes. 
Merkmale genannt, die bei Aussagen zur Kompetenz und Lebensqualität von älteren Menschen mit Behinderung zu berücksichtigen sind und die aus diesem Grunde auch als konstitutiv für erfolgreiches Altern gewertet werden:

1. Gesundheitsstatus

2. Wahrgenommene Lebenssituation

3. Die Möglichkeit mitverantwortlichen Lebens, das heißt, die Möglichkeit, sowohl zur Gemeinschaft als auch zur Gesellschaft aktiv beizutragen.

Im Arbeitskreis von Schalock wurde ein Fragebogen zur Erfassung der Lebensqualität bei älteren Menschen mit geistiger Behinderung entwickelt (Schalock, 1997). Dieser Fragebogen umfasst 40 Items, die sich auf die von der Person selbst eingeschätzten Grade der

a) Selbstständigkeit

b) Produktivität

c) Integration in die Gemeinschaft

d) Zufriedenheit

beziehen. Dieser Fragebogen wurde in der Klientel des ,Mid-Nebraska Mental Retardation Service Program' eingesetzt und diente als Grundlage für die jährlich erfolgende Evaluation des Programms. Eine Analyse von 21 60jährigen und älteren Personen ergab, dass im Laufe eines fünfjährigen Beobachtungszeitraums die selbst bewertete Lebensqualität um 3 Prozent zurückging - und mit leichten Rückgängen in den Graden der Selbstständigkeit, der Produktivität und der Integration in die Gemeinschaft. Zugleich fand sich in diesem Zeitraum eine leichte Zunahme in der selbst eingeschätzten Zufriedenheit (Schalock, 1997).

In Tabelle 1 ist eine von Schablock et al. (1999) erarbeitete Aufstellung von Dimensionen der Lebensqualität sowie von Indikatoren der einzelnen Dimensionen wiedergegeben, die - wie die Autoren auf der Grundlage einer größeren Anzahl von empirischen Studien bei älteren Menschen belegen können - zentral für die Erfassung von Lebensqualität bei Behinderung sind. Vor allem sind diese Dimensionen und Indikatoren für eine Evaluation von Programmen geeignet, die für Menschen mit Behinderungen entwickelt wurden. 


\begin{tabular}{|c|c|}
\hline Dimension & Indicators \\
\hline Emotional well-being & $\begin{array}{l}\text { Freedom from fear } \\
\text { Spirituality } \\
\text { General mood } \\
\text { Self-concept } \\
\text { Freedom from anxiety }\end{array}$ \\
\hline Interpersonal relationships & $\begin{array}{l}\text { Family } \\
\text { Friends } \\
\text { Neighbours } \\
\text { Activity personnel } \\
\text { Caregivers }\end{array}$ \\
\hline Material well-being & $\begin{array}{l}\text { Home / Residence } \\
\text { Money } \\
\text { Personal possessions } \\
\text { Clothing } \\
\text { Insurance }\end{array}$ \\
\hline Personal development & $\begin{array}{l}\text { Educational } \\
\text { Activities } \\
\text { Intellectual } \\
\text { Reading materials } \\
\text { Helping others }\end{array}$ \\
\hline Physical well-being & $\begin{array}{l}\text { Physical health } \\
\text { Food } \\
\text { Health care } \\
\text { Medication level } \\
\text { Energy level }\end{array}$ \\
\hline Self-determination & $\begin{array}{l}\text { Decisions about daily activities } \\
\text { Decisions about what I eat and drink } \\
\text { Decisions about how I spend time } \\
\text { Personal opinions } \\
\text { Personal goals }\end{array}$ \\
\hline Social inclusion & $\begin{array}{l}\text { Accepted by cohabitants } \\
\text { Accepted by staff } \\
\text { Receiving help from cohabitants } \\
\text { Receiving help from staff } \\
\text { Participation }\end{array}$ \\
\hline
\end{tabular}


Rights

Privacy
Input into decisions
Protection of possessions
Compliance with legal and human rights
Safety

Tabelle 1: Dimensionen und Indikatoren der Lebensqualität (aus: Schalock et al., 1999)

\subsection{Zentrale Kategorien innovativer Konzepte der Behindertenhilfe}

Mit den „Fachlichen Leitlinien und Empfehlungen“ des Hessischen Sozialministeriums und des Landeswohlfahrtsverbandes Hessen zur Zukunft der Behindertenhilfe wird das Ziel verfolgt, Anregungen zur Entwicklung innovativer Konzepte in der Behindertenhilfe zu geben. Unternimmt man den Versuch, die zentralen Kategorien dieser Leitbilder und Empfehlungen herauszuarbeiten, so sind zunächst drei Bereiche zu nennen, um die sich diese Leitbilder und Empfehlungen gruppieren:

1. Wohnen

2. Tagesgestaltung

3. Übergang von der Arbeit in den Ruhestand (auch im Sinne der individuellen Planung)

Darüber hinaus werden in den Leitbildern und Empfehlungen grundlegende Orientierungen sichtbar, die sich in folgenden fünf Kategorien zusammenfassen lassen:

1. Planung von Angeboten - und zwar regional wie überregional.

2. Flexibilität - und zwar sowohl bei der Planung als auch bei der Umsetzung von Angeboten sowie bei der Ansprache von Menschen mit Behinderung.

3. Übergänge zwischen verschiedenen Angeboten - vor allem zwischen jenen der Behindertenhilfe und der Altenhilfe.

4. Entscheidungskompetenz und Wahlrecht - dies vor allem mit Blick auf den Wohn- und Lebensort, auf die Gestaltung des Alltags sowie auf die Art der Begleitung, der Förderung, der Betreuung, der Unterstützung und der Pflege.

5. Beratung - mit dem Ziel, die Entscheidungskompetenz sowie das Wahlrecht nachhaltig zu stützen.

Die in den Leitbildern und Empfehlungen entwickelten Handlungsempfehlungen lassen sich wie folgt zusammenfassen:

1. Vorbereitung und Begleitung des Übergangs von der Arbeit in den Ruhestand

2. Erhaltung der individuellen Selbstorganisation durch ambulante Dienste

3. Sicherstellung ambulanter Pflegeleistungen

4. Ausbau des Betreuten Wohnens

5. Weiterentwicklung der Tagesstrukturierung und von Freizeitangeboten 
6. Förderung des Wohnens in altersheterogenen und altershomogenen Gruppen

7. Einrichtung von Wohngruppen

8. Einrichtung von Wohngruppen mit pflegerischem Betreuungsschwerpunkt

\subsection{Entwicklung innovativer Konzepte aus der Perspektive der Institutionen}

Es wurden Mitarbeiterinnen und Mitarbeiter aus 45 Einrichtungen der Behindertenhilfe dazu befragt, in welchen Bereichen der Behindertenhilfe sie Handlungsbedarf sehen, um damit einen Beitrag zur Förderung der Kompetenz und Lebensqualität bei älteren Menschen mit Behinderung zu leisten.

Die Antworten der Experten zentrierten sich um die folgenden sieben Bereiche:

1. Verstärkte Kompetenzorientierung (anstelle einer einseitigen Defizitorientierung) in der Arbeit mit älteren Menschen mit geistiger Behinderung

2. Vorbereitung auf den Berufsaustritt sowie Unterstützung beim Berufsaustritt, Ermöglichung eines allmählichen (nicht abrupten) Übergangs in die nachberufliche Zeit, Ausbau tagesstrukturierender Angebote

3. Vermehrte Berücksichtigung individueller Bedürfnisse, Kompetenzen und Interessen

4. Entwicklung spezifischer Angebote für Menschen mit seelischer Behinderung

5. Verstärkter Ausbau des betreuten Wohnens sowie von Wohngruppen in der Gemeinde, wobei diese Angebote als Ergänzung, nicht als Alternative zu stationären Einrichtungen zu verstehen sind

6. Vorbereitung der Familienangehörigen auf einen möglichen Umzug der Angehörigen mit Behinderung in eine stationäre Einrichtung, in bereutes Wohnen oder in eine Wohngruppe

7. Entwicklung einer engeren Kooperation zwischen Behindertenhilfe und Altenhilfe

Die zu den einzelnen Bereichen getroffenen Aussagen, in denen sich der wahrgenommene Handlungsbedarf widerspiegelt, seien nachfolgend zusammenfassend dargestellt.

\section{Themenbereich 1:}

\section{Vermehrte Kompetenzorientierung in der Arbeit mit älteren Menschen}

Die Einrichtungen der Behindertenhilfe sollen sich auch in der Arbeit mit älteren Menschen sehr viel stärker als heute an Kompetenzmodellen orientieren und die einseitige Orientierung an Defizitmodellen aufgeben. Diese Aussage gilt zum einen für Bildungsangebote, die auch ältere Menschen ansprechen müssen, zum anderen für die Förderung der Kompetenz im Alltag, zum dritten für Maßnahmen, die auf eine Stärkung der sozialen Teilhabe zielen, zum vierten für Maßnahmen der Aktivierung, die sich am Leitbild der Rehabilitation orientieren sollten. 
Es wird kritisch angemerkt, dass sich noch zu viele Einrichtungen an den Defizitmodellen des Alters orientieren und ältere Menschen mit Behinderung im Alter nicht ausreichend fordern und fördern. Durch die Unterschätzung der Lern- und Veränderungsfähigkeit im Alter werden Entwicklungspotenziale dieser Lebensphase nicht ausreichend ausgeschöpft.

\section{Themenbereich 2: \\ Übergang in den Ruhestand, tagesstrukturierende Angebote im Alter}

1. Der Übergang in den Ruhestand muss begleitet werden - zu nennen sind als Beispiel die gemeinsamen Nachmittage in einer Tagesstätte für ältere Menschen, in Wohnheimen wird eine Tagesstruktur angeboten, Finanzierung des Werkstattplatzes über das 65. Lebensjahr hinaus wird auf der Grundlage von Vergütungsvereinbarungen übernommen; anzustreben ist die Entwicklung eines kontinuierlichen Freizeit- und Bildungsangebots

2. Nach Ausscheiden aus der Werkstatt ist Verbleib in der vertrauten Umgebung wichtig

3. Es sollten tagesstrukturierende Angebote in der Nähe von Wohneinrichtungen geschaffen werden - notwendig ist der flächendeckende Ausbau solcher Angebote

4. Tagesstätten und den alltagsgestaltenden Angeboten kommt wachsende Bedeutung zu

5. Die Tagesstruktur in kleinen Wohneinheiten ist weiter zu entwickeln; Altentagesstätten und gemeindenahe Angebote müssen geschaffen werden

6. Freizeitangebote unter Einbeziehung ehrenamtlicher Helfer sind bedeutsam und müssen ausgebaut werden

7. Für die Tagesstruktur sind noch keine praktikablen Lösungsversuche entwickelt worden - eine enge Kooperation der Werkstattträger mit den Wohnheimträgern ist notwendig

Themenbereich 3:

Stärkere Beachtung der individuellen Bedürfnisse und Kompetenzen bei der Entwicklung von Angeboten

1. ,Präventive“ Beratung mit dem Ziel, Berührungsängste gegenüber Wohnheimen möglichst weit abzubauen - Freizeit- und Urlaubsangebote in Wohnheimen, Kurzzeitpflege

2. Über die Bedürfnisse von älteren Menschen mit Behinderungen ist zu wenig bekannt

3. Unterstützende Hilfen können Fertigkeiten erweitern

4. Vergleiche behinderte Menschen nicht mit pflegebedürftigen Menschen, sondern vergleiche sie mit nicht behinderten Menschen

5. Die Bedürfnisse von Menschen mit körperlicher Behinderung müssen bei der Entwicklung von Angeboten stärkere Beachtung finden 
6. Besondere Berücksichtigung der Lebenssituation behinderter Frauen. Bei der Pflege ist behinderten Frauen weibliche Assistenz zur Verfügung zu stellen

7. Ausbau des Case Management, um Menschen mit Behinderung und ihre Angehörigen besser begleiten und betreuen zu können

\section{Themenbereich 4: \\ Kooperation zwischen Behindertenhilfe und Altenhilfe (Vernetzung, Integration)}

1. Vernetzung zwischen Behindertenhilfe, Pflegediensten, (ambulanter, teilstationärer und stationärer) Altenhilfe und Infrastruktur in den Gemeinden

2. Kooperation zwischen Altenhilfe und Behindertenhilfe kann nur auf regionaler Ebene erfolgen - Überlegungen zur Einrichtung einer entsprechenden Regionalkonferenz werden angestellt; für die Strukturentwicklung - mit einer engeren Kooperation zwischen der Behinderten- und der Altenhilfe - sind solche Regionalkonferenzen wichtig

3. Die Eingliederungshilfe bietet mehr als die Pflegeversicherung

4. Integration von Sozialhilfeleistungen und Pflegeversicherungsleistungen

5. Aufrechterhaltung der Versorgungsverträge für Pflegeabteilungen in Einrichtungen der Behindertenhilfe

6. Angebote der Altenhilfe sollten geöffnet werden für Menschen mit Behinderung

7. Pflegerische Leistungen gehören bei Menschen mit Behinderung zur Eingliederungshilfe

8. Die fachlichen Anforderungen bei entstehendem Pflegebedarf sind ungeklärt

\section{Themenbereich 5: Angebote für Menschen mit seelischer Behinderung}

1. Es sind auch mit Blick auf psychisch erkrankte Menschen Übergänge vom ambulanten in den stationären Bereich zu schaffen

2. Es sind Tagesstättenangebote für psychisch erkrankte Menschen zu schaffen

3. Vernetzungen im Sinne eines gemeindepsychiatrischen Verbundes sind wichtig - die Hilfeleistungen der älter werdenden seelisch behinderten Menschen sollen an bereits existierende oder neu aufzubauende gemeindepsychiatrische Angebote angebunden werden, um auf diese Weise eine gemeindenahe Versorgung zu ermöglichen (Betreuung am bisherigen Lebensort)

4. Belegungskonferenzen sind notwendig, auf deren Grundlage für Menschen mit seelischer Behinderung ein Versorgungsnetz geschaffen werden kann, welches sich auf individuelle Bedarfe und Bedürfnisse einstellen kann

5. Entwicklung von Konzepten für Wohnheime für Menschen mit seelischer Behinderung, die auch bei körperlichen Einbußen den Verbleib am Wohnort ermöglichen 
6. Pflegekassen betrachten Wohneinrichtungen für Menschen mit seelischer Behinderung nicht als Wohnort, sondern als Einrichtung; aus diesem Grunde wird nur ein monatlicher Festbetrag gezahlt

\section{Themenbereich 6: Wohnen}

1. Das Betreute Wohnen (Wohnen im Verbund) ist auszubauen; die Flexibilisierung bestehender Wohnangebote ist notwendig; neue Einrichtungen müssen nicht geschaffen werden; Formen betreuten Wohnens sind für alle Gruppen behinderter Menschen zu schaffen

2. Bildung von Wohngruppen mit Schwerpunkt ,Pflege` wird vorgenommen

3. In Heimen sollten verschiedene Wohngruppen eingerichtet werden - doch sind zwei Fragen ungeklärt: (a) Wer übernimmt die Betreuung? Inwieweit sind die Berufsgruppen für die spezifischen Betreuungsaufgaben qualifiziert? (b) In welcher Trägerschaft stehen die Einrichtungen, wer ist für deren Finanzierung verantwortlich?

4. Persönliche Assistenz und unterstütztes Wohnen

\section{Themenbereich 7: Kommunale Perspektive}

1. Die kommunale Perspektive ist zu stärken - sie wird bislang zu wenig beachtet

2. Die Übernahme von Verantwortung der Kommunen für die Weiterentwicklung bestehender Angebote sowie für die Planung neuer Angebote ist vielfach zu gering

3. Der Austausch zwischen Kommunen ist zu stärken, ebenfalls die Vernetzung zwischen den Regionen - Regionalkonferenzen sind auf kommunaler Ebene auszurichten; nur wird diese Forderung praktisch nicht umgesetzt, da keiner die Initiative ergreift

4. Eine einheitliche Haltung der kommunalen Spitzenverbände ist nicht erkennbar

5. Zeitdruck und Regelungsbedarf werden unterschätzt - in Kommunen wird vielfach die Notwendigkeit zusätzlicher Angebote nicht gesehen oder es wird streng nach bisherigen Vorgaben gearbeitet, eine Flexibilisierung der Angebote findet nicht statt

6. Bedarfsanalysen und Planungskonferenzen sind bedeutsam: Eine regionalspezifische Bedarfskalkulation ist zu leisten; in diesem Kontext kommt Prognosen des Pflegebedarfs großes Gewicht zu

7. Streit um Kostenzuständigkeit bildet eine Barriere der Umsetzung

8. Klare Kompetenzen und Zuständigkeiten der Träger - dies ist auch unter dem Aspekt verstärkter und effektiver Kooperation in der Kommune wichtig.

Bedeutsam erscheint hier vor allem die Tatsache, dass zahlreiche Anforderungen an die Kommune gestellt werden. In der Diskussion über die Zukunft der Behinder- 
tenhilfe wird die kommunale Perspektive vielfach nicht ausreichend beachtet. Folgt man den Ergebnissen der Befragung, so ist die Mitverantwortung der Kommunen für die Behindertenhilfe zu stärken, darüber hinaus wird die Bedeutung einer engeren Kooperation von Institutionen für die Versorgungsqualität hervorgehoben. Die Vernetzung von Angeboten wird als große Herausforderung für die Zukunft gewertet; eine Aufgabe der Kommunen wird darin gesehen, Anstöße zu dieser Vernetzung zu geben.

Auch in einem weiteren thematischen Bereich setzen die Ergebnisse der Befragung neue Akzente. Angesprochen ist hier der Bereich der Begleitung, Betreuung, Förderung, Unterstützung und Pflege älterer Menschen mit seelischer Behinderung. Keine der drei Gruppen - Menschen mit körperlicher, mit geistiger, mit seelischer Behinderung - darf benachteiligt werden; bei der Entwicklung von Konzepten ist in gleicher Weise auf alle drei Gruppen zu achten. Die Tatsache, dass in den Befragungen ausführlich auf die Situation der älteren Menschen mit seelischer Behinderung eingegangen und großer Handlungsbedarf gerade in Bezug auf die Versorgung dieser Gruppe konstatiert wurde, zeigt, dass in Zukunft noch mehr Gewicht auf diese Personengruppe gelegt werden muss. Diese scheint - folgt man den Aussagen in den Stellungnahmen - in der Diskussion von Konzepten und Angeboten nicht selten vernachlässigt zu werden.

Die in den Leitbildern und Empfehlungen wie auch in den Befragungen sichtbar werdenden Kategorien, mit denen die Interventionskonzepte für Menschen mit Behinderung beschrieben werden, vermitteln ein modernes Bild der Behindertenhilfe.

Dieses moderne Bild der Behindertenhilfe lässt sich am besten mit den drei folgenden Kategorien charakterisieren:

- Selbstständigkeit (im Sinne möglichst weiter Unabhängigkeit bei der Ausführung von Aktivitäten des täglichen Lebens)

- Selbstverantwortung (im Sinne möglichst weiter Selbstbestimmung bei der Gestaltung des Alltags, im Sinne von Entscheidungskompetenz und Wahlfreiheit, im Sinne der bewussten Auseinandersetzung mit der eigenen Person, ihren Werten, ihren Interessen, ihren Bedürfnissen: Wer bin ich? Was möchte ich tun?)

- Mitverantwortung (im Sinne möglichst weiter sozialer Partizipation an der Gesellschaft und an der Kultur, im Sinne des Engagements für andere Menschen)

\section{Entwicklungsperspektiven}

In der Behindertenhilfe - dies gilt sowohl für die Diskussion in Deutschland als auch für die Diskussion im internationalen Raum - wird vermehrt Gewicht auf Möglichkeiten zur sozialen Teilhabe (Partizipation) gelegt. Neben der Fähigkeit zur Vorbereitung und zum Treffen von Entscheidungen (Entscheidungskompetenz) sowie der Wahlfreiheit nehmen Möglichkeiten zur sozialen Teilhabe den breitesten Raum in der Diskussion neuer Konzepte ein. Dabei wird zum einen die Bedeutung des Wohnens für die soziale Teilhabe unterstrichen: Das Betreute Wohnen und das 
Wohnen im Verbund sind zentrale Elemente des Wohnens in der Gemeinde, durch die zur vermehrten sozialen Teilhabe beigetragen wird (wie auch zur Förderung von Selbstständigkeit und Selbstverantwortung). Zudem akzentuieren stationäre Einrichtungen der Behindertenhilfe die engen Beziehungen zwischen einer Einrichtung und der Gemeinde, um auf diese Weise soziale Teilhabe zu fördern und soziale Segregation abzubauen. Neben dem Wohnen wird der Gestaltung der sozialen Beziehungen große Bedeutung für die soziale Teilhabe beigemessen. Dabei ist es wichtig, dass sich die Beziehungen nicht allein auf Menschen mit Behinderung beschränken, sondern auch Beziehungen zu Menschen ohne Behinderung umfassen. In den USA gibt es zahlreiche ehrenamtliche Initiativen, die darauf zielen, ältere Menschen aus Einrichtungen der Behindertenhilfe und ältere Menschen ohne Behinderung, die Bildungseinrichtungen besuchen oder am Vereinsleben aktiv teilnehmen, zusammenzubringen, sodass (für beide Gruppen!) ein erkennbarer Zuwachs an sozialer Teilhabe möglich wird.

In vielen Einrichtungen der Behindertenhilfe ist ein hohes Maß an Kreativität in Bezug auf die Entwicklung von Konzepten für ältere Menschen mit Behinderung erkennbar. Die Grundlage dieser Konzepte bilden dabei die Kategorien: Selbstständigkeit (im Sinne der Fähigkeit, die Aktivitäten des täglichen Lebens selbständig ausführen zu können), Selbstverantwortung (im Sinne der Autonomie und Entscheidungskompetenz) und Mitverantwortung (im Sinne der sozialen Teilhabe). Es handelt sich hier vor allem um jene Einrichtungen, die bereits über Erfahrungen in der Arbeit mit älteren Menschen, bei denen Behinderung besteht, verfügen, und vor dem Hintergrund dieser Erfahrungen Aussagen über die Lern- und Veränderungspotenziale im Alter treffen können. - Das Verständnis der Situation von Menschen mit Behinderung ist somit nicht mehr (allein) von dem Gedanken der Fürsorge geprägt, sondern (auch) von dem Gedanken der Autonomie, der Kompetenz sowie der aktiven Teilhabe an Gemeinschaft. Der Austritt aus dem Beruf ist als eine Phase des Übergangs zu verstehen, in der sich wieder vermehrt die Frage nach Möglichkeiten der selbstständigen, selbstverantwortlichen und mitverantwortlichen Lebensführung stellt. Wie müssen die Förderkonzepte beschaffen sein, um auch nach Austritt aus dem Beruf die Fähigkeit und Bereitschaft des Individuums zur Selbstständigkeit, Selbstverantwortung und Mitverantwortung zu erhalten? Dabei ist zu bedenken, dass von der Berufstätigkeit vielfach kompetenzerhaltende und kompetenzfördernde Anregungen ausgehen, die nach Austritt aus dem Beruf fortfallen. Die Einrichtungen der Behindertenhilfe sind nun in besonderer Weise gefordert, Konzepte zur Erhaltung von Kompetenz anzubieten. Zentrale Bedeutung gewinnen hier Angebote zur Tagesstrukturierung, zur sozialen Teilhabe sowie zum Wohnen (betreutes Wohnen, Wohnen in Wohngruppen). In der Weiterentwicklung bestehender Angebote sehen die Einrichtungen der Behindertenhilfe eine zentrale Zukunftsaufgabe. Der demographische Wandel führt in Einrichtungen der Behindertenhilfe zu neuen Anforderungen.

Die Zunahme des durchschnittlichen Lebensalters der Bewohnerschaft erfordert neue Akzentsetzungen im Hinblick auf heilpädagogische Angebote. Diese lassen sich charakterisieren im Sinne: 
a) der Erhaltung von Fähigkeiten und Interessen, die Menschen in früheren Lebensjahren ausgebildet haben

b) der Erschließung von neuen Möglichkeiten sozialer und kultureller Teilhabe

c) der Erhaltung von Selbstständigkeit und Selbstverantwortung auch im Falle eintretender funktioneller Einbußen.

Diese Vielfalt von Anforderungen, die Einrichtungen der Behindertenhilfe mit Blick auf ein ,gutes Leben“ im Alter zu erfüllen haben, legt die Forderung nach Aufrechterhaltung der Prinzipien der Eingliederungshilfe - und damit eines heilpädagogischen, bildungsbezogenen Konzepts - auch für ältere Menschen mit Behinderung nahe.

Die potenzielle Lern- und Veränderungsfähigkeit von älteren Menschen mit Behinderung wird erkannt - der Konzeptentwicklung liegt nicht selten ein Bildungsverständnis zugrunde, welches von der Bildbarkeit, dem Interesse an Bildung und der Notwendigkeit der Bildung über den gesamten Lebenslauf ausgeht.

In der internationalen Literatur werden vier Konzepte genannt, die für die Entwicklung der Behindertenhilfe als zentral erachtet werden:

1. Förderung von sozialer Integration, sozialer Partizipation und sozialem Engagement

2. Akzentuierung von Förderkonzepten, die von den Werten, Neigungen, Fertigkeiten und Bedürfnissen der einzelnen Person ausgehen

3. Akzentuierung der Förderung und Assistenz in der vertrauten Umwelt der Person

4. Orientierung am Leitbild des 'Ageing in place' (mit diesem Begriff wird ausgedrückt, dass Menschen auch im Alter in ihrer vertrauten Umgebung bleiben)

Dabei wird die Entwicklung der Behindertenhilfe vielfach in drei Phasen untergliedert, die unterschiedliche Akzentsetzungen der Art Dienstleistungen beschreiben:

Erste Phase: Institutionalisierung, vielfach auch Segregation. Die in der dieser Phase entwickelten Dienstleistungen orientierten sich vor allem an einem ,,medizinischen Modell“. Behinderung wurde als eine Störung verstanden, die vor allem medizinische Diagnostik, Therapie und Rehabilitation erfordere. Als geeigneter Wohnund Lebensort für Menschen mit Behinderung wurde die Institution angesehen, da nur diese die Bereitstellung der medizinischen Diagnostik, Therapie und Rehabilitation gewährleisten könne.

Zweite Phase: De-Iinstitutionalisierung und verstärkte Entwicklung des Gemeindegedankens. Die in dieser Phase entwickelten Dienstleistungen bildeten vor allem das Resultat der Normalisierungsdebatte, wie diese speziell im nordeuropäischen Raum, zum Teil auch in den Vereinigten Staaten, Kanada und Australien geführt wurde. Menschen mit Behinderung sollte die Möglichkeit gegeben werden, in gleicher Weise am Leben in der Gemeinde teilzunehmen wie Menschen ohne Behinderung. Diese Zieldefinition ging von der grundlegenden Annahme aus, dass Menschen mit Behinderung dann umfassend am gemeinschaftlichen Leben teilhaben 
können, wenn gesellschaftliche Barrieren (und hier vor allem Stereotype) abgebaut werden. Behinderung wurde nicht länger als zentrales Merkmal des Menschen gesehen. Aus diesem Grunde wurde auch nicht mehr von behinderten Menschen, sondern vielmehr von Menschen mit einer Behinderung gesprochen. Darüber hinaus wurde in dieser Phase mehr und mehr die Lern- und Veränderungsfähigkeit des Menschen mit Behinderung in den Vordergrund gestellt und die Forderung nach Entwicklung von heilpädagogischen Förderkonzepten erhoben. Man kann hier auch von einer vermehrten Kompetenz-Orientierung in der Behindertenhilfe sprechen.

Dritte Phase: Leben in der Gemeinde, aktives Mitglied der Gemeinde, individualisierende Formen der Förderung, der Unterstützung, der Assistenz. Diese Phase charakterisiert die gegenwärtige Konzeptentwicklung in der Behindertenhilfe. Die Möglichkeit des Lebens in der Gemeinde und der Aktivität in der Gemeinde ist eng verbunden mit den Wohnformen. Betreutes Wohnen oder Wohnen im Verbund bilden eine Grundlage für soziale Partizipation und soziales Engagement. Im Falle des Wohnens in einer stationären Einrichtung der Behindertenhilfe ist es notwendig, dass diese Einrichtung ausreichend in die Gemeinde integriert ist. Denn nur dann sehen sich die Bewohner ausreichend motiviert, auch wirklich am Leben in der Gemeinde teilzunehmen. - Die individualisierenden Förderkonzepte gehen von der Annahme aus, dass Menschen im Lebenslauf spezifische Fertigkeiten und Interessen entwickelt haben, die in einem biographisch orientierten Bildungs- und Förderansatz aufgegriffen werden müssen. Darüber hinaus wird die Annahme vertreten, dass die Notwendigkeit der Bildung und Förderung über den gesamten Lebenslauf hinweg besteht, somit auch ältere Menschen mit Behinderung ausdrücklich in derartige Bildungs- und Förderkonzepte einbezogen werden müssen. Die Hilfen zur Wiedereingliederung enden also nicht mit einem bestimmten Lebensalter. 